\title{
Towards IR4.0 implementation in e-manufacturing: artificial intelligence application in steel plate fault detection
}

\author{
Adeleke Abdullahi ${ }^{1}$, Noor Azah Samsudin ${ }^{2}$, Mohd Rasidi Ibrahim ${ }^{3}$, Muhammad Syariff Aripin ${ }^{4}$, \\ Shamsul Kamal Ahmad Khalid ${ }^{5}$, Zulaiha Ali Othman ${ }^{6}$ \\ ${ }^{1,2,3,4,5}$ Universiti Tun Hussein Onn Malaysia, Malaysia \\ ${ }^{6}$ National University of Malaysia, Malaysia
}

\section{Article Info}

Article history:

Received Feb 1, 2020

Revised Apr 3, 2020

Accepted Apr 17, 2020

Keywords:

Artificial intelligence

Fault detection

Machine learning

Manufacturing

\begin{abstract}
Fault detection is the task of discovering patterns of a certain fault in industrial manufacturing. Early detection of fault is an essential task in industrial manufacturing. Traditionally, faults are detected by human experts. However, this method suffers from cost and time. In this era of Industrial revolution IR 4.0, machine learning (ML) methods and techniques are developed to solve fault detection problem. In this study, three standard ML models: LR, NB, and SVM are developed for the classification problem. The experimental dataset used in this study consists of steel plates faults. The dataset is retrieved from UCI machine learning repository. Three standard evaluation methods: accuracy, precision, and recall are validated on the classification models. Logistic regression (LR) model achieved the highest accuracy and precision scores of $94.5 \%$ and 0.756 respectively. In addition, the SVM model had the highest recall score of 0.317 . The results showed the significant impact of AI/ML approach in steel plates fault diagnosis problem.
\end{abstract}

Copyright $\odot 2020$ Institute of Advanced Engineering and Science. All rights reserved.

\section{Corresponding Author:}

Adeleke Abdullahi,

Faculty of Computer Science and Information Technology,

Universiti Tun Hussein Onn Malaysia.

Email:abdul2040@yahoo.com

\section{INTRODUCTION}

A fault may be defined as an abnormal condition or defect which could lead to a failure. Early detection of fault is an essential task in industry production [1]. Fault detection (or diagnosis) [2] is the task of discovering patterns of a certain fault in industrial manufacturing. These patterns include time, location, and size among others. Over the years, manual fault detection $[3,4]$ has been the traditional way of finding faults. An expert often acquires information of working equipment, study the manual guiding the equipment maintenance, and finally diagnosed the possible causes of a certain fault. The major setbacks associated with the traditional fault detection (FD) system are timing and cost [4].

With the advancement in industrial manufacturing, the introduction of Industrial Revolution (IR) 4.0 $[5,6]$, a concept motivated from the digital revolution involving the interconnection between people and technology. In other words, IR 4.0 involves the technological expansion where several ways of demonstrating skills and abilities are discoverable in order to minimize the gap between physical, digital, and biological entities. The digital revolution in manufacturing has led to the development of technologies such as robotics, intelligent systems, human computer interaction, and additive manufacturing. These technologies have had significant impact on manufacturing. The development of autonomous fault detection system could greatly help in achieving the tasks previously done manually with less computational time and cost. The automated system could employ the concept and techniques of artificial intelligence (AI) and machine learning in executing the dedicated tasks. 
The field of machine learning (ML) [7] focuses on the study that gives AI system the capability to improve its performance (decision making) over a time period through acquiring new knowledge and skills (learning/training), as well as its ability to reorganize the existing knowledge based on the newly acquired knowledge. The basic concept of ML is typically the goal of modeling machines for critical decision-making purposes. One of the most important and widely studied technique in artificial intelligence and machine learning is data mining [8-10], which is the problem of discovering hidden patterns from data. Data mining (DM) techniques has been extensively studied and applied to solve real world problems such as prediction (or classification), clustering, imaging, cybersecurity, $e$-business, manufacturing, and sports. Furthermore, conventional among machine learning algorithms often implemented in ML tasks include: naïve bayes (NB) [11], decision trees (J48) [12], neural networks [13-19], support vector machines (SVM) [20-23], and $k$-nearest neighbour $(k$-NN) [24-28].

As aforementioned, timely detection of faults is an important task in industrial manufacturing. The steel industry is one of the important areas which has fault detection problem. This study focused on the application of ML approach in steel plates fault detection. To achieve this, standard predictive algorithms (also called classifiers) are implemented. These classifiers include logistic regression, naïve bayes, and support vector machines. The experimental results are validated using three conventional performance metrics: accuracy, precision, and recall.

In [29] experimental work, Support Vector Machines (SVM) method was applied for fault diagnosis. The results showed an effective reduction in the dimensionality of the sample space with high classification accuracy at lower computational time. The findings in [1] highlighted some of the significant importance of fault detection (FD) in steel manufacturing such as errors reduction, minimize loss, and accurate decision-making. The study employed predictive models: Random Forest, Artificial Neural Network (ANN), and SVM, in training and testing industrial data. The models were used to solve some of the complex problems associated with industrial manufacturing. The results showed Random Forest achieved overall highest accuracy of $77.8 \%$.

Furthermore, [30] presented in their research work an intelligent system based on ANN for extracting useful information and predict quality features in multistage manufacturing process. The input data include temperature, material conditions, force, and vibration while the output data include coordinative measurements. The results showed the proposed system achieved high degree of accuracy in predicting the end product quality. From other existing related works include [31-40].

This paper presents the potential of machine learning approach in steel plates faults detection problem. In this work, standard machine learning algorithms are applied. The study employed three ML classification algorithms (or classifiers). These classifiers include Logistic Regression (LR), Naïve Bayes (NB), and Support Vector Machines (SVM). Section 2 documents the methodology employed in executing the classification task.

\section{METHODS AND MATERIALS}

Experimental Dataset: The Steel plates' faults dataset is used in the experimental work. The dataset (obtained from the UCI machine learning repository) is one of the available datasets utilized to classify steel plate's faults into seven distinct types: Pastry, Z-Scratch, K-Scratch, Stains, Dirtiness, Bumps, and Other_Faults. The dataset consists of 1941 instances with distinct fault types labels. As tabulated in Table 1, each instance of the dataset has 27 independent variables and a fault type.

Table 1: Steel plate's faults dataset with class distribution and predictive attributes

\begin{tabular}{cccccc}
\hline Output class & \# of Cases & & \multicolumn{2}{c}{ Predictive attributes } \\
\hline Pastry & 158 & Attribute 1 & X_Minimum & Attribute 14 & Steel_Plate_Thickness \\
Z_Scratch & 190 & Attribute 2 & X_Maximum & Attribute 15 & Edges_Index \\
K_Scratch & 391 & Attribute 3 & Y_Minimum & Attribute 16 & Empty_Index \\
Stains & 72 & Attribute 4 & Y_Maximum & Attribute 17 & Square_Index \\
Dirtiness & 55 & Attribute 5 & Pixels_Areas & Attribute 18 & Outside_X_Index \\
Bumps & 402 & Attribute 6 & X_Perimeter & Attribute 19 & Edges_X_Index \\
Other_Faults & 673 & Attribute 7 & Y_Perimeter & Attribute 20 & Edges_Y_Index \\
& & Attribute 8 & Sum_of_Luminosity & Attribute 21 & Outside_Global_Index \\
& & Attribute 9 & Minimum_of_Luminosity & Attribute 22 & LogOfAreas \\
& & Attribute 10 & Maximum_of_Luminosity & Attribute 23 & Log_X_Index \\
& & Attribute 11 & Length_of_Conveyer & Attribute 24 & Log_Y_Index \\
& & Attribute 12 & TypeOfSteel_A300 & Attribute 25 & Orientation_Index \\
& & Attribute 13 & TypeOfSteel_A400 & Attribute 26 & Luminosity_Index \\
& & & Attribute 27 & SigmoidOfAreas \\
\hline
\end{tabular}


Classification models: The overall goal of this study is to evaluate the potential of machine learning approach in steel plate's faults detection. To achieve this, three ML classifiers: logistic regression (LR), Naïve Bayes (NB), and Support Vector Machine (SVM) will be implemented on the steel plate's faults dataset. The classifiers are trained to predict or classify the faults.

Logistic Regression (LR) [41-44] is a multivariate statistical method that can be used to evaluate the inter-relationship between dependent and independent variables. The technique works by classifying records based on values of input attributes. For each record, a probability of membership is computed for all possible output categories. The target category with the highest probability is assigned as the predicted output value. LR is an efficient approach which helps both in prediction of data classification sample as well as the calculation of the probability of classification. Given the probability of $y=1$, logistic regression model is built using (1).

$$
\operatorname{logit}(y)=C_{0}+C_{1} x_{1}+C_{2} x_{2}+\cdots+C_{n} x_{n}
$$

where $x_{1}, x_{2}, \ldots, x_{n}$ are predictions while $y$ is the output to predict. Furthermore, $\log$ it $(y)$ could be expressed as:

$$
\operatorname{logit}(y)=\ln \left(\frac{y}{1-y}\right)
$$

Naïve bayes classifier greatly simplify learning by assuming that features are independent given class and has proven effective in many practical applications, including data classification [45]. The classification method is a simple probabilistic model based on the Bayes rule. To determine the probability of a document $d$ belonging to a class $C$, the naive bayes model follows (3).

$$
P\left(C_{i} \mid d\right)=\frac{P\left(d \mid C_{i}\right) * P\left(C_{i}\right)}{P(d)}
$$

Support vector machines (SVM) algorithm is typically used for learning classification, regression, or ranking function. The algorithm works by searching a seperating hyperplane to seperate between samples with a maximal margin. The equation for hyperplane is:

$$
w^{T} x+b=0
$$

where $w$ is the weight vector and $b$ is the threshold.

Performance Metrics: The study validated the experimental results using accuracy, precision, and recall performance. These are among the conventional evaluation methods [45] used in prediction or classification. Given a confusion matrix, the accuracy, precision, and recall metrics are calculated as:

$$
\begin{aligned}
& \text { accuracy }=\frac{T P+T N}{T P+F P+T N+F N} \\
& \text { precision }=\frac{T P}{T P+F P} \\
& \text { recall }=\frac{T P}{T P+F N}
\end{aligned}
$$

where $T P=$ True positive

$T N=$ True negative

$F P=$ False positive

$F N=$ False negative

The steel plate's faults dataset is divided into training set and testing set using percentage ratio 70:30\% respectively. The training set is used to build and estimate the model parameters, while the test set is used to independently validate the trained prediction model. As earlier mentioned, the dataset consists of 1941 instances distinctly labeled into one of 7 fault types: Pastry, Z-Scratch, K-Scratch, Stains, Dirtiness, Bumps, and Other_Faults. Each of the 1941 instances owns 27 independent variables and one fault type. The experimental dataset is explored for incorrect, inconsistent, and missing data. The predictive attributes are of numeric type: flag or range, while the target class is of type nominal. 


\section{EXPERIMENTAL RESULTS AND ANALYSIS}

The classification performance of the predictive models was evaluated using three conventional performance measures: classification accuracy, precision, and recall which are described in (4), (5), and (6) respectively. Figures 1 to 3 show the interface of the experiment using the LR, NB, and SVM on Azure Machine Learning software. The metrics are calculated using the confusion matrix table comprising of true positive $(T P)$, true negative $(T N)$, false positive $(F P)$, and false negative $(F N)$. The classifiers were implemented using the entire steel plate's faults dataset. The classification results obtained established the significant influence of machine learning approach to faults detection problem.

Working with machine learning classification models in the steel plate's faults detection problem had promising results as could be seen from the result in Table 2. Remarkably, the three classification methods achieved above $90 \%$ accuracy result. Logistic Regression model had the overall accuracy result of 94.5\% while naïve bayes had the result of 94\% accuracy. This possibly is due to the sensitivity of the methods to the features (attributes set). Furthermore, LR had the highest precision value while Support vector machine had the highest recall value. This shows how difficult it is to conclude on which particular model is the best. Classification models are highly competitive with varying strengths and weaknesses. Overall, the experimental results showed the significant impact of applying AI/ML approach in detecting faults in industrial manufacturing.

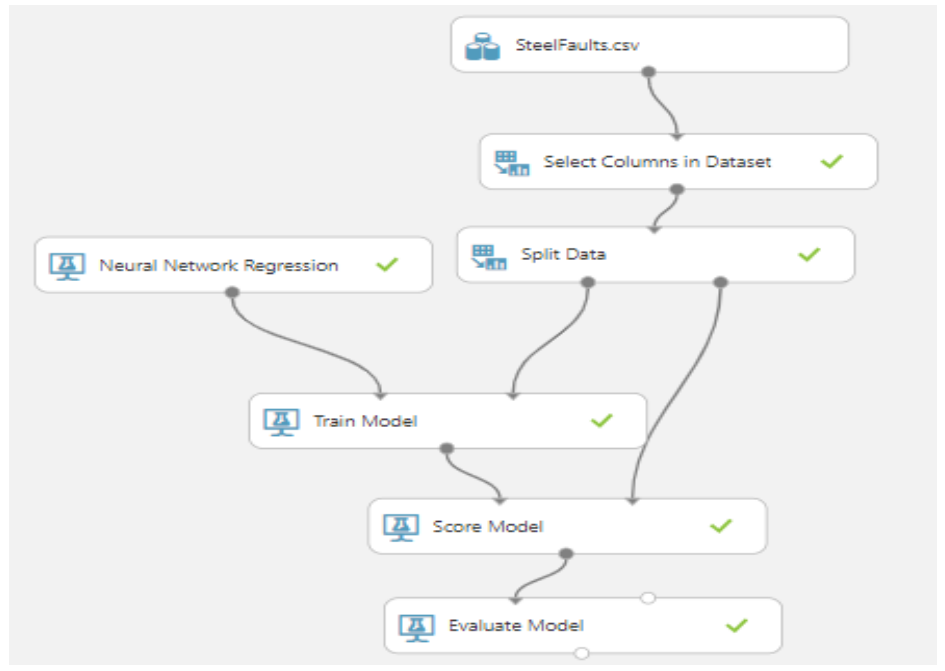

Figure 1. Interface of experiment using logistic regression on azure machine learning software

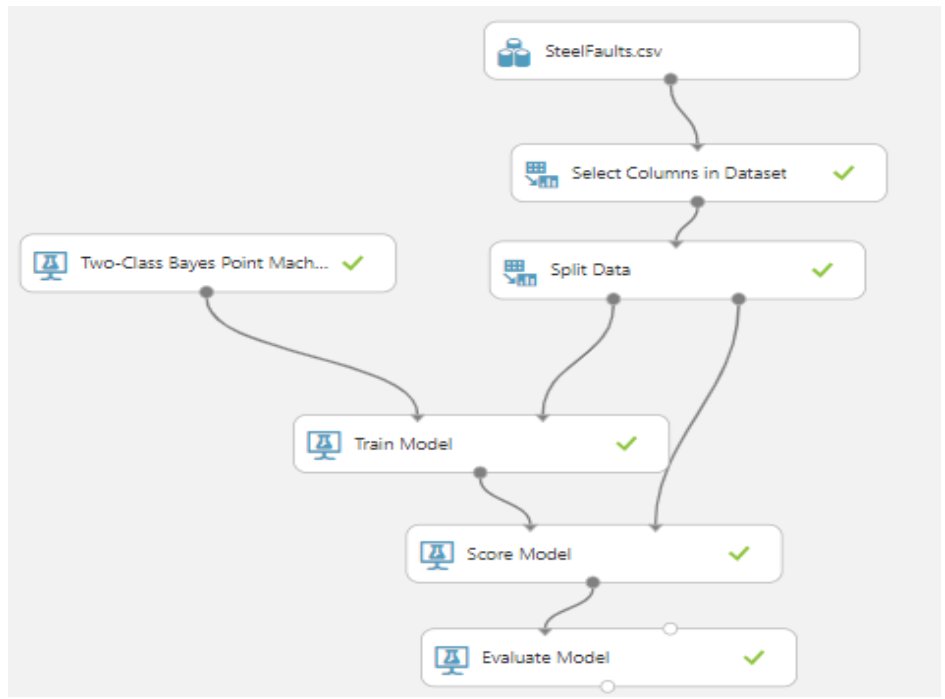

Figure 2. Interface of experiment using naïve bayes on azure machine learning software 


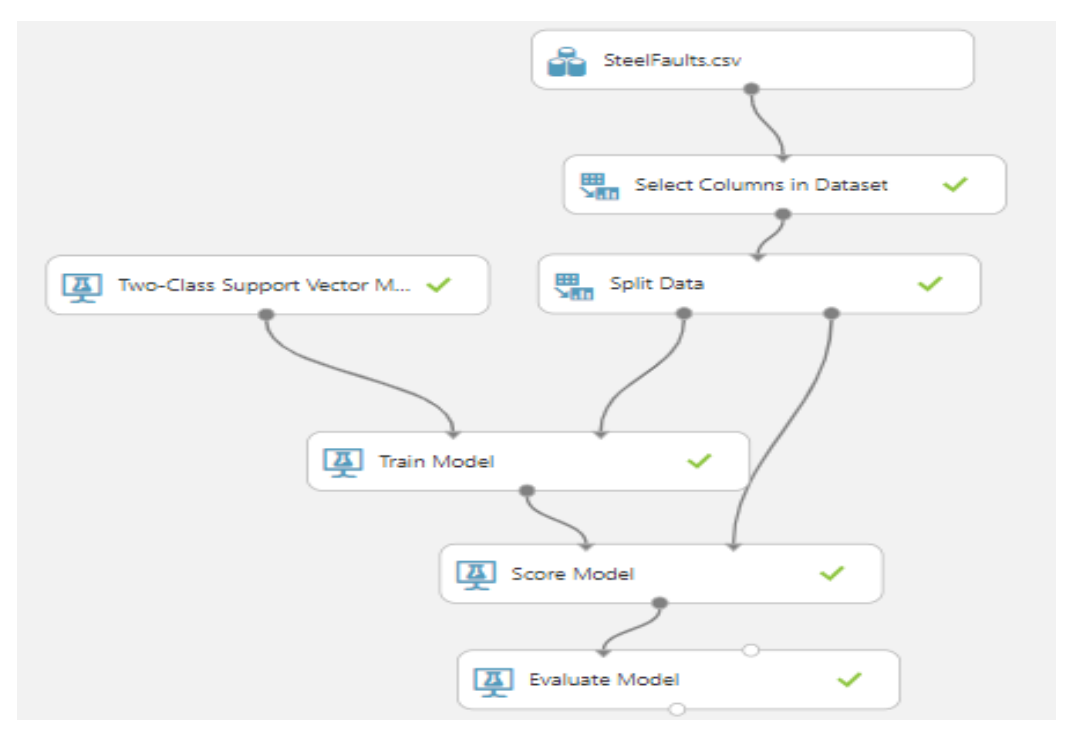

Figure 3. Interface of experiment using support vector machine on azure machine learning software

Table 2. Classification performance of the predictive models on the steel plate's faults dataset

\begin{tabular}{cccc}
\hline Classification models & ACC $(\%)$ & Precision & Recall \\
\hline LR & 94.5 & 0.756 & 0.2 \\
NB & 94 & 0.688 & 0.268 \\
SVM & 94.2 & 0.684 & 0.317 \\
\hline
\end{tabular}

\section{CONCLUSION}

This study has successfully implemented the comparative analysis on the automated fault diagnostic application in steel manufacturing problem domain. We found that the AI or machine learning techniques have the potential to detect problems in steel manufacturing industry. Such findings may be extended to other applications in manufacturing industry, towards fulfilling Industry Revolution 4.0 requirements.

\section{ACKNOWLEDGEMENTS}

The authors would like to thank the Universiti Tun Hussein Onn Malaysia for supporting this research under Multidisciplinary Research Grant Scheme, Vot No. H511.

\section{REFERENCES}

[1] T. Nkonyana, "Performance Evaluation of Data Mining Techniques in Steel Manufacturing Industry," Procedia Manufacturing, vol. 35, pp. 623-628, 2019.

[2] L. Yao, et al., "A novel intelligent method for fault diagnosis of electric vehicle battery system based on wavelet neural network," Journal of Power Sources, vol. 453, pp. 1-12, 2020.

[3] S. Nasiri, et al., "Fracture mechanics and mechanical fault detection by artificial intelligence methods: A review," Engineering Failure Analysis, vol. 81, pp. 270-293, 2017.

[4] B. Song, et al., "Fault detection and diagnosis via standardized k nearest neighbor for multimode process," Journal of the Taiwan Institute of Chemical Engineers, vol. 106, pp. 1-8, 2020.

[5] A. Schumacher, et al., "Roadmapping towards industrial digitalization based on an industry 4.0 maturity model for manufacturing enterprises," in $12^{\text {th }}$ CIRP Conf. on Intelligent Computation in Manufacturing Engineering, vol. 79, pp. 409-414, 2019.

[6] W. S. Alaloul, et al., "Industrial Revolution 4.0 in the construction industry: challenges and opportunities for stakeholders," Ain Shams Engineering Journal, vol. 11, no. 1, pp. 225-230, 2020.

[7] A. O. Adeleke, et al., "A Group-Based Feature Selection Approach to Improve Classification of Holy Quran Verses," in R. Ghazali et al. (eds.), Recent Advances on Soft Computing and Data Mining, Advances in Intelligent Systems and Computing 700, pp. 282-297, 2018.

[8] A. M. Jabbar, et al., "An improved ACS algorithm for data clustering," Indonesian Journal of Electrical Engineering and Computer Science, vol. 17, no. 3, pp. 1506-1515, 2020.

[9] H. R. Esmaeel, "Analysis of classification learning algorithms," Indonesian Journal of Electrical Engineering and Computer Science, vol. 17, no. 2, pp. 1029-1039, 2020. 
[10] W. F. W. Yaacob, "Supervised data mining approach for predicting student performance," Indonesian Journal of Electrical Engineering and Computer Science, vol. 16, no. 3, pp. 1584-1592, 2019.

[11] A. Adeleke, et al., "Automating Quranic Verses Labeling Using Machine Learning Approach," Indonesian Journal of Electrical Engineering and Computer Science, vol. 16, no. 2, pp. 925-931, 2019.

[12] A. Adeleke, et al., "A Two-Step Feature Selection Method for Quranic Text Classification," Indonesian Journal of Electrical Engineering and Computer Science, vol. 16, no. 2, pp. 730-736, 2019.

[13] S. A. Mohamed, et al., "A review on data clustering using spiking neural network (SNN) models," Indonesian Journal of Electrical Engineering and Computer Science, vol. 15, no. 3, pp. 1392-1400, 2019.

[14] W. Lumchanow and S. Udomsiri, "Image Classification of malaria using hybrid algorithms: Convolutional neural network and method to find appropriate k for k-nearest neighbor," Indonesian Journal of Electrical Engineering and Computer Science, vol. 16, no. 1, pp. 382-388, 2019.

[15] N. Ab. Wahab, et al., "Artificial neural network based technique for energy management prediction," Indonesian Journal of Electrical Engineering and Computer Science, vol. 17, no. 1, pp. 94-101, 2020.

[16] Y. Park and H. Yang, "Convolutional neural network based on an extreme learning machine for image classification," Neurocomputing, vol. 339, pp. 66-76, 2019.

[17] X. Qi, et al., "Applying Neural Network-Based Machine Learning to Additive Manufacturing: Current Applications, Challenges, and Future Perspective," Engineering, vol. 5, no. 4, pp. 721-729, 2019.

[18] Z. Chiba, et al., "Intelligent approach to build a Deep Neural Network based IDS for cloud environment using combination of machine learning algorithms," Computers \& Security, vol. 86, pp. 291-317, 2019.

[19] F. Jia, et al., "A neural network constructed by deep learning technique and its application to intelligent fault diagnosis of machines," Neurocomputing, vol. 272, pp. 619-628, 2018.

[20] O. W. Chuan, et al., "Fault classification in smart distribution network using support vector machine," Indonesian Journal of Electrical Engineering and Computer Science, vol. 18, no. 3, pp. 1148-1155, 2020.

[21] M. Fikri and R. Sarno, "A comparative study of sentiment analysis using SVM and SentiWordNet," Indonesian Journal of Electrical Engineering and Computer Science, vol. 13, no. 3, pp. 902-909, 2019.

[22] M. Tanaskuli, et al., "Ozone prediction based on support vector machine," Indonesian Journal of Electrical Engineering and Computer Science, vol. 17, no. 3, pp. 1461-1466, 2020.

[23] A. F. H. Alharan, et al., "A cluster-based feature selection method for image texture classification," Indonesian Journal of Electrical Engineering and Computer Science, vol. 14, no. 3, pp. 1433-1442, 2019.

[24] W. Li, et al., "Boosted k-nearest neighbor classifiers based on fuzzy granules," Knowledge-Based Systems, vol. 195, pp. 1-13, 2020.

[25] P. Skryjomski, et al., "Speeding up k-nearest neighbor classifier for large-scale multi-label learning on GPUs," Neurocomputing, vol. 354, pp. 10-19, 2019.

[26] Q. Dai, et al., “A novel clustering algorithm based on the natural reverse nearest neighbor structure," Information Systems, vol. 84, pp. 1-16, 2019.

[27] B. Fan, et al., "Efficient nearest neighbor search in high dimensional hamming space," Pattern Recognition, vol. 99, p. 107082, 2020.

[28] J. Gou, et al., "Locality constrained representation-based k-nearest neighbor classification," Knowledge-Based Systems, vol. 167, pp. 38-52, 2019.

[29] Y. Tian, et al., "Steel plates fault diagnosis in the basis of support vector machines," Neurocomputing, vol. 15, no. 1, pp. 296-303, 2015.

[30] M. Papananias, et al., "An Intelligent Metrology Informatics Systems based on Neural Networks for Multistage Manufacturing Processes," in $17^{\text {th }}$ CIRP Conference on Modelling of Machining Operations, vol. 82, pp. 444-449, 2019.

[31] F. Assad, et al., "A Framework to predict energy related key performance indicators of manufacturing systems at early design phase," in 52 ${ }^{\text {nd }}$ CIRP Conference on Manufacturing Systems, vol. 81, pp. 145-150, 2019.

[32] O. G. Miller and V. Gandhi, "A survey of modern exogenous fault selection and diagnosis methods for swarm robotics," Journal of King Saud University - Engineering Sciences, pp. 1-11, 2019.

[33] R. J. O'Brien, et al., "A pattern recognition system based on acoustic signals for fault detection on composite materials," European Journal of Mechanics A/Solids, vol. 64, pp. 1-10, 2017.

[34] J. Malek and T. N. Desai, "A systematic literature review to map literature focus of sustainable manufacturing," Journal of Cleaner Production, vol. 256, pp. 1-20, 2020.

[35] Q. Hu, et al., "A rotating machinery fault diagnosis method based on multi-scale dimensionless indicators and random forests," Mechanical Systems and Signal Processing, vol. 139, pp. 106609-106630, 2020.

[36] Y. Lei, et al., "Applications of machine learning to machine fault diagnosis: A review and roadmap," Mechanical Systems and Signal Processing, vol. 138, pp. 106587-106625, 2020.

[37] Y. He, et al., "Fault correction of algorithm implementation for intelligentized robotic multipass welding process based on finite state machines," Robotics and Computer Integrated Manufacturing, vol. 59, pp. 28-35, 2019.

[38] Y. Xue, et al., "Multi-fault diagnosis of rotating machinery based on deep convolution neural network and support vector machine," Measurement, vol. 156, pp. 107571-107577, 2020.

[39] Y. Lu and Y. Li, "A novel fault diagnosis method for circuit breakers based on optimized affinity propagation clustering," International Journal of Electrical Power and Energy System, vol. 118, pp. 1-9, 2020.

[40] Y. Liu, et al., "Weighted contribution rare of nonlinear output frequency response functions and its application to rotor system fault diagnosis," Journal of Sound and Vibration, vol. 460, pp. 1-20, 2019.

[41] Q. Sun, et al., "Improved mapping and understanding of desert vegetation-habitat complexes from intraanual series of spectral endmember space using cross-wavelet transform and logistic regression," Remote Sensing of Environment, vol. 236, pp. 1-14, 2020. 
[42] A. M. Bianco, et al., "Robust wald-type methods for testing equality between two population regression parameters: A comparative study under the logistic model," Computational Statistics and Data Analysis, vol. 142, pp. 1-16, 2020.

[43] J. Feng, et al., "Comparison between logistic regression and machine learning algorithms on survival prediction of traumatic brain injuries," Journal of Critical Care, vol. 54, pp. 110-116, 2019.

[44] D. B. Ionescu, et al., "On the prediction of geoeffectiveness of CMEs during the ascending phase of SC24 using a logistic regression method," Journal of Atmospheric and Solar Terrestrial Physics, vol. 193, pp. 1-6, 2019.

[45] A. Adeleke, et al., "Comparative Analysis of Text Classification Algorithms for Automated Labelling of Quranic Verses," International Journal on Advance Science, Engineering and Information Technology, vol. 7, no. 4, pp. 1419-1427, 2017.

\section{BIOGRAPHIES OF AUTHORS}

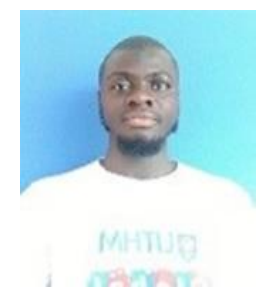

Abdullahi Adeleke is a $\mathrm{PhD}$ student at Faculty of Computer Science and Information Technology, Universiti Tun Hussein Onn Malaysia (UTHM) since March 2018. He had his master degree in Information Technology (MIT) at UTHM. His research interests include ICT, machine learning, data mining, document (text) classification, and feature selection techniques.

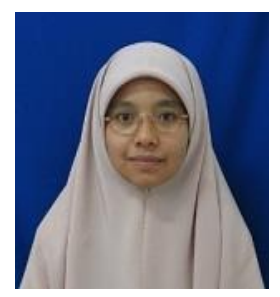

Noor Azah Samsudin is currently an Associate Professor at Faculty of Computer Science and Information Technology, Universiti Tun Hussein Onn Malaysia (UTHM). She received her bachelor degree in Computer Science from University Missouri-Columbia in 1996. Then, she accomplished her master degree at National University of Malaysia. She received her PhD from The University of Queensland, Australia. Her research interest includes machine learning, data mining and ICT applications in industry and education.

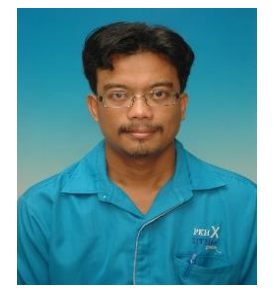

Mohd Rasidi Ibrahim is currently an Associate Professor at Faculty of Mechanical Engineering, Universiti Tun Hussein Onn Malaysia (UTHM). He received his Diploma in Tool and Die Technology, Production Technology from German Malaysia Institute, Kuala Lumpur, Malaysia. Then, he pursued his Bachelor degree in Manufacturing Engineering at Leeds Metropolitan University, United Kingdom. He then completed his PhD in Mechanical Engineering at University of Brunel, West London. In total, he also has over five years of industrial experience with well-known companies in Malaysia and United Kingdom. His research focus on advance and precision manufacturing machining.

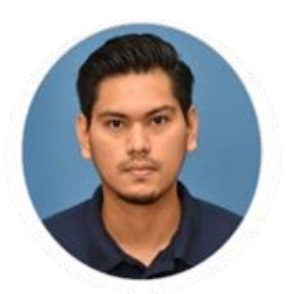

Muhammad Syariff bin Aripin was a student at Faculty of Computer Science and Information Technology, Universiti Tun Hussein Onn Malaysia (UTHM) since 2015. He received his Diploma in Information Technology at Politeknik Muadzam Shah, Malaysia and eventually, he accomplished his Bachelor Degree in Information Technology at Universiti Tun Hussein Onn Malaysia (UTHM). Currently, he is appointed as research assistant for Artificial Intelligence applications for various projects at UTHM.

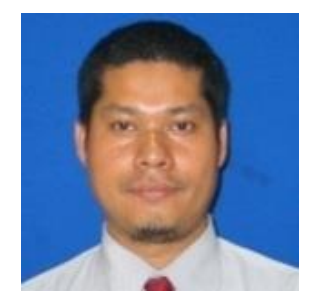

Shamsul Kamal Ahmad Khalid is a senior lecturer at Faculty of Computer Science and Information Technology, Universiti Tun Hussein Onn Malaysia (UTHM) since March 2004. He received his bachelor degree in Computer Science from New York University in 1995. Then, he accomplished his master degree at National University of Malaysia. He received his $\mathrm{PhD}$ from Universiti Tun Hussein Onn Malaysia. His research interest includes information security, watermarking, steganography, and network security.

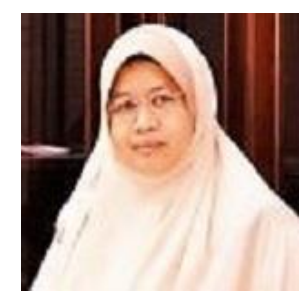

Zulaiha Ali Othman is currently an Associate Professor at National University of Malaysia. Her research interest is on artificial intelligence, Big Data, and optimization algorithms in various problem domain including network intrusion, human talent, climate change and pollution. 